\title{
A Novel Robust Controller of Drug Dosage for Cancer Chemotherapy
}

\author{
Vineet Gali \\ Georgia Institute of Technology, Georgia \\ galivineet678@gmail.com
}

\begin{abstract}
One of the dangerous death-causing diseases called cancer can be cured by exploiting a renowned technique called chemotherapy. Nowadays, to find the optimal drug rate injection the controller-based technique is exploited therefore, the treatment has increased sufficiently. In this paper, a new robust controller which influences the dosage of drug besides the parameter estimation is presented in this paper. A new adaptive square-root unscented Kalman filter (ASr-UKF)is developed in this work. In reality, by exploiting the existing difference function, the error is calculated and it is deployed for the updating procedure of the proposed method in the conventional methods. On the basis of the prior error function, the updating process is performed while scaled to a nonlinear environment. Moreover, to update the process, the scaling factor is developed that includes enhancement in the historical error. At last, the developed controller performance is considered with the conventional methods that entail the suitable effect of drug dosage injection on normal, tumor and immune cells. In addition, it is seen that the adopted model possesses the capability to calculate tumor cells by means ofa superior accuracy rate
\end{abstract}

Keywords:Cancer, Chemotherapy, Controller, Drug Dosage, Error Function

\begin{tabular}{ll} 
Nomenclature & \\
\hline Abbreviations & Descriptions \\
\hline NAC & Neoadjuvant Chemotherapy \\
ASr-UKF & Adaptive Square-Root Unscented Kalman Filter \\
RTs & Responding Tumors \\
GBD & Global Burden Disease \\
CNNs & Deep convolutional neural networks \\
pCR & Pathological Complete Remission \\
VTE & Venous Thromboembolism \\
NAC & Neoadjuvant Chemotherapy \\
US & ultrasound \\
\hline
\end{tabular}

\section{Introduction}

In women, breast cancer is represented as persistent cancer, which leads to cancer-associated death globally. The systematic treatment is the Preoperative NAC which is exploited for patients with locally highly developed breast cancer. By suggesting intracellular damage, the NAC treatment minimizes the tumor size which set up the breast protecting treatment. It can be related to considerably superior eventfree survival and superior complete survival particularly in patients by means of triple-negative and HER2+ breast cancer. Nevertheless, because of diverse histopathological and molecular kinds of cancer reaction to NAC therapy can be complicated to consider [1].

For example, at the time of the treatment there may be no obvious minimization of huge size, still in scenario of positively RTs [2]. Now and then, the therapy might outcome in tumor fragmentation creating the evaluation of mass challenges. Untimely response prediction to chemotherapy is very important for health patients for various causes. Initially, in the scenario of non- RTs, it set up switching to diverse therapy. Subsequently, it is significant to halt the ineffectual treatment as soon as probable due to the chemotherapy's toxic side effects [4]. 
Breast cancer is yet a public health issue, in spite of the immense development and several treatments. In accordance with the most recent GBD report, breast cancer is yet an important general cancer type among females and the next general cancer type amid all kinds of cancers in the United States and the globe. Patients who undergo surgery frequently require chemotherapy to minimize the breast cancer tumor size and minimize the difficulties subsequent to the surgery. Chemotherapy, in spite of aiding to heal disease subsequent to the surgery, particularly in long run related with several physical and psychological difficulties [3].In the physical dimension chemotherapy integrated with the surgery minimizes the immunity, body functions that causes anemia. Additionally, if the psychological dimension raises depression, anxiety and the life quality of $\mathrm{BC}$ patients is reduced. The very important chemotherapy side effect is fatigue which causes to depress in the long phase.

At present, deep learning techniques are attaining the impetus for the characterization of breast mass in Ultrasonic imaging. Aforesaid data-driven techniques have the ability to robotically procedure US data to learn to resolve examined tasks. To distinguish benign and malignant breast masses exploits the Deep CNN swhich was very effectively employed in numerous studies [10], [11].

For example, a deep learning-based technique was superior to 4 radiologists in the scenario of the breast mass classification. For the segmentation and detection of breast masses, the CNN was exploited. Generally, in US imaging, the datasets are very tiny to model the deep learning techniques from scratch. To extract the generic features, the pre-trained CNNs are used, which is related to the attendance of several image characteristics, associated with the color blobs and to object edges.

The main objective of this research is to propose a new robust controller which controls dosage of drug together by means of the parameter estimation. Moreover, a new ASr-UKF is developed and updating procedure is performed based upon prior error function, via scaled to a nonlinear environment. Furthermore, a scaling factor is developed so that the improvement in historical error is considered for the updating procedure. At last, the adopted model performance is compared with the conventional models that exhibit the effect of drug dosage injection on immune, normal, as well as tumor cells.

\section{Literature Review}

In 2021, SalehoddinBouya et al [1], worked on the general psychological reactions the person exhibits at the time of the treatment and the diagnosis. In this work, to ascertain the efficiency of the treatment self-care enlightening involvement on minimizing hopelessness in women with breast cancer experiencing the postmastectomy chemotherapy was also presented. In the simulation analysis, the experimentation was analyzed on ninety women who have breast cancer. In 2019, XIN FENG et al [2], examined the prospect of PCR prediction by exploiting merely the initial three treatments nodal sizes. Optimal feature integration for every breast cancer subkind was examined from real nodal sizes of the initial three treatments and nodal sizes of subsequent three treatments forecasted from those of 1 stthree ones. In 2019, Michal Byra et al [3], developed deep learning in US imaging on the basis of technique for untimely NAC response prediction. To model the response prediction techniques, the transfer learning with deep CNNs was exploited. The helpfulness of two transfer learning methods has experimented. Initially, on the ImageNet dataset,t a CNN pre-trained was exploited. Next, double transfer learning was exploited. In 2021, Florian Moik et al [4], worked on the patients with cancer experiencing the chemotherapy were at thrombocytopenia risk. The co-occurrence of cancer-related thrombocytopenia, and VTE, was a recurrent difficulty in patients with cancer. Particularly in definite tumor entities at maximum VTE risk, chemotherapeutic agents with possessions of myelosuppressive were kind of normal of care. In 2021, Amanda K. Arrington et al [5], worked on the R0 resection for pancreatic cancer was taking into consideration the standard of care, however, it was not attained. Here, R1/R2 resection results evaluated with chemotherapy were presented. From NCDB 2010-2017 the examination was performed regarding the Stage two pancreatic cancers which undergo R1/R2 surgery without/with neoadjuvant chemotherapy.

\section{Non-linear Mathematical Formulation for Chemotherapy}

The main objective of this research is to present a novel nonlinear control technique explicitly important robust over uncertainties of the parameter. Additionally, the performance of the system is validated by considering the chemotherapy of the minimum order model. The proposed approach considers the interfering of tumor cells with immune and normal cells. From eq. (1) to (3) exhibits the non-linear technique in that the, $\mathrm{T}(\mathrm{n}) \mathrm{ID}(\mathrm{n})$ and $\mathrm{D}(\mathrm{n})$ symbolizes a number of normal, immune, andtumor cells at a time $\mathrm{n}$, respectively. 


$$
\overrightarrow{\mathrm{ID}}=\mathrm{st}+\frac{\rho \mathrm{ID} \cdot \mathrm{T}}{\alpha+\mathrm{T}}-\mathrm{q}_{1} \mathrm{ID} \cdot \mathrm{T}-\mathrm{t}_{1} \mathrm{ID}-\mathrm{y}_{1} \mathrm{u}_{1} \mathrm{ID}
$$

Moreover, drug injections are on basis of considered control inputs. The $u_{1}(n), u_{2}(n)$ and $u_{3}(n)$ signifies ad chemotherapy drug effect. This technique presumes akind ofimmune cell, using a kinetic process tumor size can be reduced.

$$
\overrightarrow{\mathrm{T}}=\mathrm{ra}_{1} \mathrm{~T}\left(1-\mathrm{c}_{1} \mathrm{~T}\right)-\mathrm{q}_{2} \mathrm{ID} \cdot \mathrm{T}-\mathrm{q}_{3} \mathrm{TD}-\mathrm{y}_{2} \mathrm{u}_{2} \mathrm{~T}
$$

Moreover, the technique comprises immunity cells whereas the growth is enthused using the tumor existence namely T-cells. By exploiting a chemotherapeutic drug it is assumed that the whole-cell populations are evaded with varied proportions.

$$
\overrightarrow{\mathrm{D}}=\mathrm{ra}_{2} \mathrm{D}\left(1-\mathrm{c}_{2} \mathrm{D}\right)-\mathrm{q}_{4} \mathrm{TD}-\mathrm{y}_{3} \mathrm{u}_{3} \mathrm{D}
$$

Lymph nodes, and Bone marrow, are particular resources that could model a constant source for ID cells, st as shown in the firstvariable of eq. (1). $\frac{\rho \mathrm{ID} \cdot \mathrm{T}}{\mathrm{T}}$ denotes saturation function with positive conditions which include $\rho$ and $\alpha$ and this constraint directly shows immune cells are processed using tumor cells. The contest between tumors and immune cellsis indicated by $q_{1}$ ID T which reasons the loss of immune cells. Moreover, $\mathrm{y}_{1} \mathrm{u}_{1} \mathrm{ID}$ indicates the immune cell loss because of drug injection and $\mathrm{t}_{1} \mathrm{ID}$ indicates that immune cells obtain insolvent at $t_{1}$ natural death rate.

$\mathrm{ra}_{1} \mathrm{~T}\left(1-\mathrm{c}_{1} \mathrm{~T}\right)$ indicates improvement of the tumor cell populationas stated in eq. (2) which is shown as logistic variablewith $\mathrm{t}_{1}^{-1}$ maximum performing potential and $\mathrm{ra}_{1}$ growth rate. The disputeamidrate of growth and death is stated using the logistic growth variable [8]. $\mathrm{q}_{2} \mathrm{ID} \cdot \mathrm{T}$ signifies competition amidtumor and immune cells that ensues in tumor cell loss. As same as, the dispute between normal and tumor cells are indicated as $q_{3}$ TD that reasons the tumor cell lossand $y_{2} u_{2} R$ indicates tumor cell loss because of drug injection.

Eq. (3), $\mathrm{ra}_{2} \mathrm{D}\left(1-\mathrm{c}_{2} \mathrm{D}\right)$ signifies population normal cell growth as a logistic techniquewith ra 2 growth rate as well $t_{2}^{-1}$. Moreover, $\mathrm{q}_{4} \mathrm{RD}$ indicates the conflicts amid normal and tumor cellswhich origin normal cell loss and $\mathrm{y}_{3} \mathrm{u}_{3} \mathrm{D}$ indicatesnormal cell loss due todrug injection. For that reason, $\mathrm{y}_{1}, \mathrm{y}_{2}$ and $\mathrm{y}_{3}$ indicateschemotherapy effect on evading cell population.

\section{System Modeling}

For the third-order nonlinear technique, a robust adaptive control system is explained. The controllers aspire is to track system conditions(tumor, immune, and normal cells) by means of particular best values. Therefore, to attain this purpose, biological cell volumes (immune, tumor, and normal) are estimated against their best values and error signals are generated, and drug dosages are recommended on the basis of this. Moreover, to model a robust system against parameter vagueness, parameters are calculated and used in the control loop. Using eq. (1), (2), and (3), the third-order model of tumor is rephrased as in Eq. (4) which is furthermore reorganized in Eq. (5).

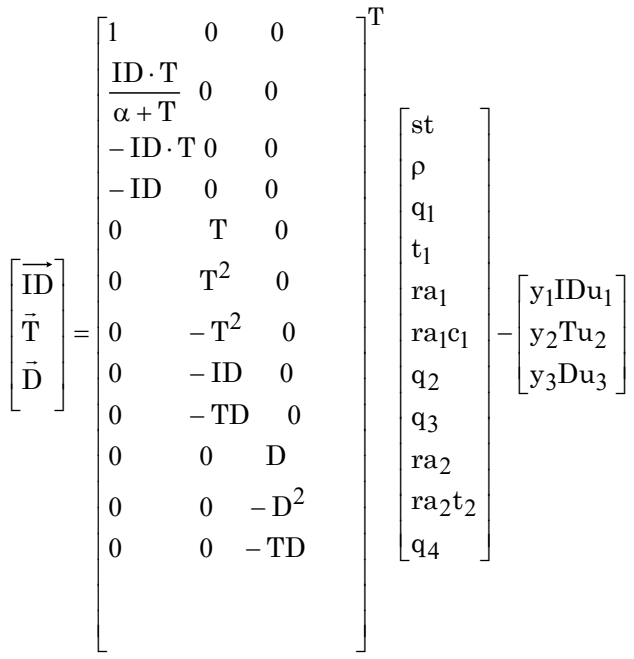


Eq.(6)exhibitsregression vectors. To consider uncertainty in technique, constraints are substituted by means of estimated values and parameter vectors are attained as stated in Eq. (7).

$$
\begin{aligned}
& \left.\left[\begin{array}{l}
\mathrm{u}_{1} \\
\mathrm{u}_{2} \\
\mathrm{u}_{3}
\end{array}\right]=\left[\begin{array}{lll}
\frac{1}{\mathrm{~T}} & 0 & 0 \\
\frac{\mathrm{T}}{\alpha+\mathrm{T}} & 0 & 0 \\
-\mathrm{T} & 0 & 0 \\
-1 & 0 & 0 \\
\frac{-\mathrm{ID}}{\mathrm{ID}} & 0 & 0 \\
0 & 1 & 0 \\
0 & -\mathrm{T} & 0 \\
0 & \mathrm{ID} & 0 \\
0 & -\mathrm{T} & 0 \\
0 & \frac{-\mathrm{T}}{\mathrm{T}} & 0 \\
0 & 0 & 1 \\
0 & 0 & -\mathrm{D} \\
0 & 0 & -\mathrm{T} \\
0 & 0 & \frac{-\mathrm{D}}{\mathrm{D}}
\end{array}\right]^{\mathrm{T}}\right]^{\mathrm{T}}\left[\begin{array}{l}
\frac{\mathrm{st}}{\mathrm{y}_{1}} \\
\frac{\rho}{\mathrm{y}_{1}} \\
\frac{\mathrm{q}_{1}}{\mathrm{y}_{1}} \\
\frac{\mathrm{t}_{1}}{\mathrm{y}_{1}} \\
\frac{1}{\mathrm{y}_{1}} \\
\frac{\mathrm{ra}}{\mathrm{y}_{2}} \\
\frac{\mathrm{ra}_{1} \mathrm{c}_{1}}{\mathrm{y}_{2}} \\
\frac{\mathrm{q}_{2}}{\mathrm{y}_{2}} \\
\frac{\mathrm{q}_{3}}{\mathrm{y}_{2}} \\
\frac{1}{\mathrm{y}_{2}} \\
\frac{\mathrm{ra}_{2}}{\mathrm{y}_{3}} \\
\frac{\mathrm{ra}_{2} \mathrm{c}_{2}}{\mathrm{y}_{3}} \\
\frac{\mathrm{q}_{4}}{\mathrm{y}_{3}} \\
\frac{1}{\mathrm{y}_{3}}
\end{array}\right] \\
& \mathrm{G}_{1}=\left[\begin{array}{lllll}
\frac{1}{\mathrm{ID}} & \frac{\mathrm{T}}{\alpha+\mathrm{T}} & -\mathrm{T} & -1 & \frac{-\mathrm{ID}}{\mathrm{ID}}
\end{array}\right] \\
& \mathrm{G}_{2}=\left[\begin{array}{lllll}
1 & -\mathrm{T} & -\mathrm{ID} & -\mathrm{D} & \frac{-\mathrm{T}}{\mathrm{T}}
\end{array}\right] \\
& \mathrm{G}_{3}=\left[\begin{array}{llll}
1 & -\mathrm{D} & -\mathrm{T} & \frac{-\mathrm{D}}{\mathrm{D}}
\end{array}\right]
\end{aligned}
$$

Based on this, the conditions (normal, tumor, and immune cells) track their equivalent optimal values and which are derived based on Eq. (8) to formulate a stable model.

$$
\begin{aligned}
& \bar{\theta}_{1}=\left[\begin{array}{lllll}
\overline{\mathrm{st}} & \bar{\rho} \\
\overline{\bar{y}_{1}} & \frac{\overline{\mathrm{y}}_{1}}{\overline{\mathrm{y}}_{1}} & \frac{\overline{\mathrm{t}}_{1}}{\overline{\mathrm{y}}_{1}} & \frac{1}{\overline{\mathrm{y}}_{1}} & \frac{\overline{\mathrm{y}}_{1}}{{ }^{\mathrm{T}}}
\end{array}\right]^{\mathrm{T}} \\
& \bar{\theta}_{2}=\left[\frac{\overline{\mathrm{ra}}_{1}}{\mathrm{y}_{2}} \frac{\overline{\mathrm{ra}}_{2} \overline{\mathrm{c}}_{2}}{\mathrm{y}_{2}} \frac{\overline{\mathrm{c}}_{2}}{\overline{\mathrm{y}}_{2}} \frac{\overline{\mathrm{c}}_{3}}{\overline{\mathrm{y}}_{2}} \frac{1}{\overline{\mathrm{y}}_{2}}\right]^{\mathrm{T}}
\end{aligned}
$$

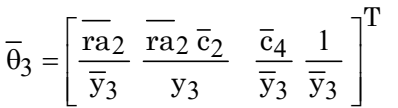

In Eq. (8), $\gamma_{1}, \gamma_{2}$ and $\gamma_{3}$ indicates positive constants. $\overrightarrow{\mathrm{ID}}_{\mathrm{t}}$ and $\mathrm{ID}_{\mathrm{t}}$ represents derivativesof the immune cell and optimal values. $\vec{D}_{t}$ and $\mathrm{D}_{\mathrm{t}}$ indicates derivatives of normal cells and desired values, $\mathrm{T}_{\mathrm{t}}$ and $\overrightarrow{\mathrm{T}}_{\mathrm{t}}$ signifies tumor cells optimal values and its derivatives. Eq. (9) can beobtained, by substituting Eq. (8) in Eq. (6),

$$
\begin{aligned}
& \overrightarrow{\mathrm{ID}}=\overrightarrow{\mathrm{ID}}_{\mathrm{t}}-\gamma_{1}\left(\mathrm{ID}-\mathrm{ID}_{\mathrm{t}}\right) \\
& \overrightarrow{\mathrm{T}}=\overrightarrow{\mathrm{T}}_{\mathrm{t}}-\gamma_{2}\left(\mathrm{~T}-\mathrm{T}_{\mathrm{t}}\right) \\
& \overrightarrow{\mathrm{D}}=\overrightarrow{\mathrm{D}}_{\mathrm{t}}-\gamma_{3}\left(\mathrm{D}-\mathrm{D}_{\mathrm{t}}\right)
\end{aligned}
$$




$$
\begin{aligned}
& \mathrm{G}_{1}\left(\overline{\mathrm{ID}}_{\mathrm{t}}-\gamma_{1}\left(\mathrm{ID}-\mathrm{ID}_{\mathrm{t}}\right), \mathrm{ID}, \mathrm{D}, \mathrm{T}\right)=\left[\frac{1}{\mathrm{ID}} \frac{\mathrm{T}}{\alpha+\mathrm{T}}-\mathrm{T}-1 \frac{-\overline{\mathrm{ID}}_{\mathrm{t}}-\gamma_{1}\left(\mathrm{ID}-\mathrm{ID}_{\mathrm{t}}\right)}{\mathrm{IM}}\right]
\end{aligned}
$$

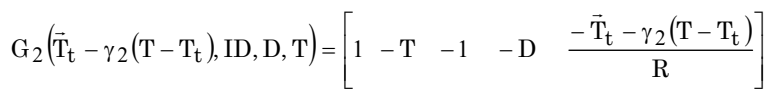

$$
\begin{aligned}
& \mathrm{G}_{3}\left(\overrightarrow{\mathrm{D}}_{\mathrm{t}}-\gamma_{3}\left(\mathrm{D}-\mathrm{D}_{\mathrm{t}}\right), \mathrm{ID}, \mathrm{D}, \mathrm{T}\right)=\left[\begin{array}{llll}
1 & -\mathrm{D} & -\mathrm{T} & \frac{-\mathrm{D}_{\mathrm{t}}-\gamma_{3}\left(\mathrm{D}-\mathrm{D}_{\mathrm{t}}\right)}{\mathrm{D}}
\end{array}\right]
\end{aligned}
$$

In Eq. (10), the control principle is represented based on the regressor modelof system formulation, in that $\bar{\theta}_{1}, \bar{\theta}_{2}$ and $\bar{\theta}_{3}$ indicates assessing vector constraints. Moreover, in eq. (11), adaptation law toevaluate vector parameter are stated, in that $\Gamma_{1}, \Gamma_{2}$ and $\Gamma_{3}$ indicates the symmetric positive definite constant measures. As stated in Eq. (12), error vectors of immune, normal, and tumor cells are statedas $\overleftrightarrow{\mathrm{D}}, \overleftrightarrow{\mathrm{ID}}$ and $\overleftrightarrow{\mathrm{T}}$ , correspondingly.

$$
\begin{aligned}
& \left\{\begin{array}{l}
\mathrm{u}_{1}=\mathrm{G}_{1} \bar{\theta}_{1} \\
\mathrm{u}_{2}=\mathrm{G}_{2} \bar{\theta}_{2} \\
\mathrm{u}_{3}=\mathrm{G}_{3} \bar{\theta}_{3}
\end{array}\right. \\
& \overline{\overline{\theta_{1}}}=\overleftrightarrow{\mathrm{ID}} \cdot \mathrm{ID} \cdot \Gamma_{1} \mathrm{G}_{1}^{\mathrm{T}} \operatorname{sign}\left(\mathrm{y}_{1}\right) \\
& \overline{\overline{\theta_{2}}}=\stackrel{\leftrightarrow}{\mathrm{T}} \cdot \mathrm{T} \cdot \Gamma_{2} \mathrm{G}_{2}^{\mathrm{T}} \operatorname{sign}\left(\mathrm{y}_{2}\right) \\
& \overline{\overline{\theta_{3}}}=\overline{\overline{\mathrm{D}}} \cdot \mathrm{D} \cdot \Gamma_{3} \mathrm{G}_{3}^{\mathrm{T}} \operatorname{sign}\left(\mathrm{y}_{3}\right) \\
& \overleftrightarrow{\mathrm{ID}}=\mathrm{ID}-\mathrm{ID}_{\mathrm{t}}
\end{aligned}
$$

\section{Proposed Adaptive Square-Root Unscented Kalman Filter}

In the lab environment, the immune cell measurement is complex and it requires certain analysis and hence a nonlinear observer is neededtoassess immune cells.

$$
\dot{y}\left(t^{\prime}\right)=f\left(y\left(t^{\prime}\right), u\left(t^{\prime}\right)\right)+w\left(t^{\prime}\right)
$$

Concerning nonlinear systems, diverse techniques are developed to approximate the nonlinearities of the system's dynamics, namely EKF and UKF. The disadvantages of these techniques are discussed and identified.

Here, initially the principles of the Sr-UKF are developed; subsequently, an adaptive version of the filter is stated that is superior to the traditional Sr-UKF in the scenario of unknown or time-varying noise distributions. The proposed method [7] is stated as below:

The initial step of the approach is the initialization. In this stage, the first values of states and the square-root of the error covariance matrix are considered as stated as below:

$$
\mathrm{z}\left(\mathrm{t}^{\prime}\right)=\mathrm{g}\left(\mathrm{y}\left(\mathrm{t}^{\prime}\right)\right)+\mathrm{v}\left(\mathrm{t}^{\prime}\right)
$$

Whereas, $\mathrm{y} \in \mathrm{R}^{\mathrm{n}}$ represents the state vector, $\mathrm{z} \in \mathrm{R}^{\mathrm{m}}$ and $\mathrm{u} \in \mathrm{R}^{\mathrm{L}}$ indicates the input and output. The square-root of error the covariance matrix $S_{k}$ is stated as below:

$$
\begin{aligned}
\hat{\mathrm{y}}_{0} & =\mathrm{E}\left[\mathrm{y}_{0}\right] \\
\mathrm{s}_{0} & =\mathrm{CH}\left(\mathrm{E}\left[\left(\mathrm{y}_{0}-\hat{\mathrm{y}}_{0}\right)\left(\mathrm{y}_{0}-\hat{\mathrm{y}}_{0}\right)^{\mathrm{T}}\right]\right)
\end{aligned}
$$

$\mathrm{CH}$ indicates the Cholesky factor and $\left(\hat{\mathrm{y}}_{\mathrm{k}-1 \mid \mathrm{k}-1}^{\mathrm{i}}\right)$ represents the sigma point.

$$
\begin{aligned}
& \mathrm{E}\left[\left(\mathrm{y}_{0}-\hat{\mathrm{y}}_{0}\right)\left(\mathrm{y}_{0}-\hat{\mathrm{y}}_{0}\right)^{\mathrm{T}}\right] \\
& \left(\hat{\mathrm{y}}_{\mathrm{k}-1 \mid \mathrm{k}-1}\right) \text { and }\left(\mathrm{s}_{\mathrm{k}-1 \mid \mathrm{k}-1}\right),\left(\hat{\mathrm{y}}_{\mathrm{k}-1 \mid \mathrm{k}-1}^{\mathrm{i}}\right)
\end{aligned}
$$

$\mathrm{L}$ indicates scaling parameter, $\alpha^{2}$ indicates weighting factor, $\lambda=\mathrm{L}\left(\alpha^{2}-1\right)$

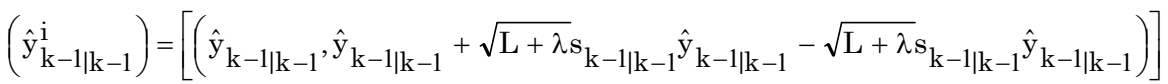

$$
\begin{aligned}
& \delta_{\mathrm{k}-1 \mid \mathrm{k}-1}^{\mathrm{i}}=\mathrm{f}\left(\hat{\mathrm{y}}_{\mathrm{k}-1 \mid \mathrm{k}-1}^{\mathrm{i}}, \mathrm{u}_{\mathrm{k}-1 \mid \mathrm{k}-1}\right), \mathrm{i}=0,1, \ldots, 2 \mathrm{~L}
\end{aligned}
$$

The time and mean update of the Cholesky factor, are computed as follows: 


$$
\hat{\mathrm{y}}_{\mathrm{k}-1 \mid \mathrm{k}-1}=\sum_{\mathrm{i}=0}^{2 \mathrm{n}} \mathrm{w}_{\mathrm{i}}^{\mathrm{m}} \delta_{\mathrm{k} \mid \mathrm{k}-1}^{\mathrm{i}}
$$

where $Q^{\prime} R^{\prime}$ indicates the return matrix $w_{i}^{c}$ and $w_{i}^{m}$ represents the weight.

$$
\begin{aligned}
& \mathrm{S}_{\mathrm{k}-1 \mid \mathrm{k}-1}=\mathrm{Q}^{\prime} \mathrm{R}^{\prime}\left(\left[\sqrt{\mathrm{w}_{\mathrm{i}}^{\mathrm{c}}}\left(\delta_{\mathrm{k} \mid \mathrm{k}-1}^{\mathrm{i}}-\mathrm{y}_{\mathrm{k} \mid \mathrm{k}-1}\right) \sqrt{\mathrm{Q}^{\prime}}\right]\right) \\
& \mathrm{S}_{\mathrm{k}-1 \mid \mathrm{k}-1}=\mathrm{CU}\left(\left[\mathrm{s}_{\mathrm{k} \mid \mathrm{k}-1}, \delta_{\mathrm{k} \mid \mathrm{k}-1}^{0}-\hat{\mathrm{y}}_{\mathrm{k} \mid \mathrm{k}-1} \mathrm{w}_{0}^{\mathrm{c}}\right]\right) \\
& \mathrm{w}_{\mathrm{i}}^{\mathrm{m}}=\frac{\lambda}{\mathrm{L}+\lambda} \\
& \mathrm{w}_{0}^{\mathrm{c}}=\frac{\lambda}{\mathrm{L}+\lambda}+\left(1-\alpha^{2}+\beta\right) \\
& \mathrm{w}_{\mathrm{i}}^{\mathrm{m}}=\mathrm{w}_{\mathrm{i}}^{\mathrm{c}}=\frac{\lambda}{2(\mathrm{~L}+\lambda)} ; \mathrm{i}=1,2, \ldots . ., 2 \mathrm{~L}
\end{aligned}
$$

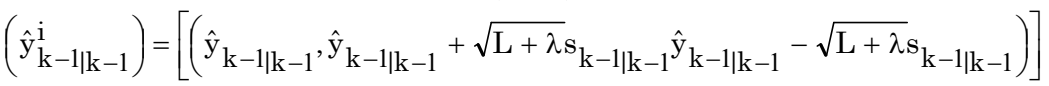

$$
\begin{aligned}
& \hat{\mathrm{y}}_{\mathrm{k}-1 \mid \mathrm{k}-1}=\sum_{\mathrm{i}=0}^{2 \mathrm{n}} \mathrm{w}_{\mathrm{i}}^{\mathrm{m}} \mathrm{y}_{\mathrm{k} \mid \mathrm{k}-1}^{\mathrm{i}} \\
& \mathrm{S}_{\mathrm{k}}^{\mathrm{zZ}}=\mathrm{QR}\left(\left[\sqrt{\mathrm{w}_{\mathrm{i}}^{\mathrm{c}}}\left(\delta_{\mathrm{k} \mid \mathrm{k}-1}^{\mathrm{i}}-\mathrm{y}_{\mathrm{k} \mid \mathrm{k}-1}\right) \sqrt{\mathrm{R}}\right]\right) \\
& \mathrm{S}_{\mathrm{k}}^{\mathrm{zZ}}=\mathrm{CU}\left(\left[\mathrm{S}_{\mathrm{k}}^{\mathrm{yy}}, \delta_{\mathrm{k} \mid \mathrm{k}-1}^{0}-\hat{\mathrm{y}}_{\mathrm{k} \mid \mathrm{k}-1} \mathrm{w}_{0}^{\mathrm{c}}\right]\right) \\
& \mathrm{P}_{\mathrm{k}}^{\mathrm{yz}}=\sum_{\mathrm{i}=0}^{2 \mathrm{n}}\left\{\mathrm{w}_{\mathrm{i}}^{\mathrm{c}}\left(\delta_{\mathrm{k} \mid \mathrm{k}-1}^{\mathrm{i}}-\hat{\mathrm{y}}_{\mathrm{k} \mid \mathrm{k}-1}\right)\left(\delta_{\mathrm{k} \mid \mathrm{k}-1}^{\mathrm{i}}-\hat{\mathrm{y}}_{\mathrm{k} \mid \mathrm{k}-1}\right)^{\mathrm{T}}\right\}
\end{aligned}
$$

The Kalman filter gain is computed using the following formulations:

$$
\begin{aligned}
& \mathrm{K}=\mathrm{P}_{\mathrm{k}}^{\mathrm{yz}} /\left(\mathrm{S}_{\mathrm{k}}^{\mathrm{zz}}\right)^{\mathrm{T}} / \mathrm{S}_{\mathrm{k}}^{\mathrm{zz}} \\
& \left(\hat{\mathrm{y}}_{\mathrm{k} \mid \mathrm{k}}^{\mathrm{i}}\right)=\hat{\mathrm{y}}_{\mathrm{k} \mid \mathrm{k}-1}^{\mathrm{i}}+\mathrm{K}_{\mathrm{k}}\left[\left(\hat{\mathrm{y}}_{\mathrm{k}}-\hat{\mathrm{y}}_{\mathrm{k}-1 \mid \mathrm{k}-1}\right)\right] \\
& \mathrm{U}=\mathrm{K}_{\mathrm{k}} \mathrm{S}_{\mathrm{k}}^{\mathrm{yy}} \\
& \mathrm{S}_{\mathrm{k} \mid \mathrm{k}}=\mathrm{CU}\left(\left[\mathrm{S}_{\mathrm{k} \mid \mathrm{k}-1}, \mathrm{U}-1\right]\right)
\end{aligned}
$$

To calculate mean and noise covariance values are calculated as follows:

$$
\begin{aligned}
& J^{*}=p[Y(k), Q, R, q, r \mid Z(k)]=\frac{p[Y(k)|Z(k), Q, R, q, r| Y(k) Q, R, q, r]}{p[Z(k)]} \\
& J=p[Y(k), Q, R, q, r] \times p[Y(k)|, Q, R, q, r| P[Q, R, q, r] \\
& \operatorname{Cov}\left[w_{i}, w_{k}\right]=0, i \neq k \\
& \operatorname{Cov}\left[v_{i}, v_{k}\right]=0, i \neq k \\
& \operatorname{Cov}\left[v_{i}, w_{k}\right]=0, i \neq k
\end{aligned}
$$


The estimation model of the developed technique is stated as follows:

\section{Pseudocode of the proposed algorithm}

Initialization $\hat{\mathrm{y}}_{0}=\mathrm{E}\left[\mathrm{y}_{0}\right]$

$$
\mathrm{S}_{0}=\mathrm{CH}\left(\mathrm{E}\left[\left(\mathrm{y}_{0}-\hat{\mathrm{y}}_{0}\right)\left(\mathrm{y}_{0}-\hat{\mathrm{y}}_{0}\right)^{\mathrm{T}}\right]\right)
$$

For all samples do

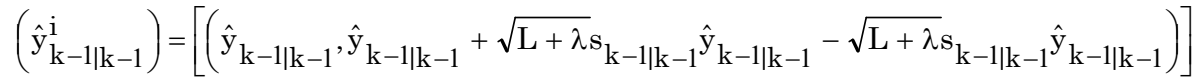

$$
\begin{aligned}
& \delta_{\mathrm{k}-1 \mid \mathrm{k}-1}^{\mathrm{i}}=\mathrm{f}\left(\hat{\mathrm{y}}_{\mathrm{k}-1 \mid \mathrm{k}-1}^{\mathrm{i}}, \mathrm{u}_{\mathrm{k}-1 \mid \mathrm{k}-1}\right), \mathrm{i}=0,1, \ldots, 2 \mathrm{~L} \\
& \hat{\mathrm{y}}_{\mathrm{k}-1 \mid \mathrm{k}-1}=\sum_{\mathrm{i}=0}^{2 \mathrm{n}} \mathrm{w}_{\mathrm{i}}^{\mathrm{m}} \delta_{\mathrm{k} \mid \mathrm{k}-1}^{\mathrm{i}} \\
& \mathrm{S}_{\mathrm{k}-1 \mid \mathrm{k}-1}=\mathrm{Q}^{\prime} \mathrm{R}^{\prime}\left(\left[\sqrt{\mathrm{w}_{\mathrm{i}}^{\mathrm{c}}}\left(\delta_{\mathrm{k} \mid \mathrm{k}-1}^{\mathrm{i}}-\mathrm{y}_{\mathrm{k} \mid \mathrm{k}-1}\right) \sqrt{\mathrm{Q}^{\prime}}\right]\right) \\
& \mathrm{S}_{\mathrm{k}-1 \mid \mathrm{k}-1}=\mathrm{CU}\left(\left[\mathrm{s}_{\mathrm{k} \mid \mathrm{k}-1}, \delta_{\mathrm{k} \mid \mathrm{k}-1}^{0}-\hat{\mathrm{y}}_{\mathrm{k} \mid \mathrm{k}-1} \mathrm{w}_{0}^{\mathrm{c}}\right]\right) \\
& \left(\hat{\mathrm{y}}_{\mathrm{k}-1 \mid \mathrm{k}-1}^{\mathrm{i}}\right)=\left[\left(\hat{\mathrm{y}}_{\mathrm{k}-1 \mid \mathrm{k}-1}, \hat{\mathrm{y}}_{\mathrm{k}-1 \mid \mathrm{k}-1}+\sqrt{\mathrm{L}+\lambda \mathrm{s}_{\mathrm{k}-1 \mid \mathrm{k}-1}} \hat{\mathrm{y}}_{\mathrm{k}-1 \mid \mathrm{k}-1}-\sqrt{\mathrm{L}+\lambda} \mathrm{s}_{\mathrm{k}-1 \mid \mathrm{k}-1}\right)\right] \\
& \hat{\mathrm{y}}_{\mathrm{k}-1 \mid \mathrm{k}-1}=\mathrm{g}\left(\delta_{\mathrm{k} \mid \mathrm{k}-1}^{\mathrm{i}}\right) \quad \hat{\mathrm{y}}_{\mathrm{k}-1 \mid \mathrm{k}-1}=\sum_{\mathrm{i}=0}^{2 \mathrm{n}} \mathrm{w}_{\mathrm{i}}^{\mathrm{m}_{\mathrm{k} \mid \mathrm{k}-1}} \delta^{\mathrm{i}}
\end{aligned}
$$

Measurement update

$$
\begin{aligned}
& \mathrm{S}_{\mathrm{k}}^{\mathrm{ZZ}}=\mathrm{Q}^{\prime} \mathrm{R}^{\prime}\left(\left[\sqrt{\mathrm{w}_{\mathrm{i}}^{\mathrm{c}}}\left(\delta_{\mathrm{k} \mid \mathrm{k}-1}^{\mathrm{i}}-\mathrm{y}_{\mathrm{k} \mid \mathrm{k}-1}\right) \sqrt{\mathrm{R}}\right]\right) \\
& \mathrm{S}_{\mathrm{k}}^{\mathrm{zZ}}=\mathrm{CU}\left(\left[\mathrm{S}_{\mathrm{k}}^{\mathrm{yy}}, \delta_{\mathrm{k} \mid \mathrm{k}-1}^{0}-\hat{\mathrm{y}}_{\mathrm{k} \mid \mathrm{k}-1} \mathrm{w}_{0}^{\mathrm{c}}\right]\right) \\
& \mathrm{P}_{\mathrm{k}}^{\mathrm{yz}}=\sum_{\mathrm{i}=0}^{2 \mathrm{n}}\left\{\mathrm{w}_{\mathrm{i}}^{\mathrm{c}}\left(\delta_{\mathrm{k} \mid \mathrm{k}-1}^{\mathrm{i}}-\hat{\mathrm{y}}_{\mathrm{k} \mid \mathrm{k}-1}\right)\left(\delta_{\mathrm{k} \mid \mathrm{k}-1}^{\mathrm{i}}-\hat{\mathrm{y}}_{\mathrm{k} \mid \mathrm{k}-1}\right)^{\mathrm{T}}\right\}
\end{aligned}
$$

States enhancement

$$
\begin{aligned}
& \mathrm{K}=\mathrm{P}_{\mathrm{k}}^{\mathrm{yz}} /\left(\mathrm{S}_{\mathrm{k}}^{\mathrm{zz}}\right)^{\mathrm{T}} / \mathrm{S}_{\mathrm{k}}^{\mathrm{zz}} \\
& \left(\hat{\mathrm{y}}_{\mathrm{k} \mid \mathrm{k}}^{\mathrm{i}}\right)=\hat{\mathrm{y}}_{\mathrm{k} \mid \mathrm{k}-1}^{\mathrm{i}}+\mathrm{K}_{\mathrm{k}}\left[\left(\hat{\mathrm{y}}_{\mathrm{k}}-\hat{\mathrm{y}}_{\mathrm{k}-1 \mid \mathrm{k}-1}\right)\right] \\
& \mathrm{U}=\mathrm{K}_{\mathrm{k}} \mathrm{S}_{\mathrm{k}}^{\mathrm{yy}} \\
& \mathrm{S}_{\mathrm{k} \mid \mathrm{k}}=\mathrm{CU}\left(\left[\mathrm{S}_{\mathrm{k} \mid \mathrm{k}-1}, \mathrm{U}-1\right]\right)
\end{aligned}
$$

Noise estimation

$$
\zeta_{\mathrm{k}}=\hat{\mathrm{y}}_{\mathrm{k}}-\hat{\mathrm{y}}_{\mathrm{k}-1 \mid \mathrm{k}-1}-\ddot{\mathrm{r}}_{\mathrm{k}}
$$

End for

\section{Results and Discussions}

In this paper, the developed control theory for cancer chemotherapy was presented. Moreover, the performance was performed by setting nominal constraints of chemotherapy as shown in this section. Furthermore, the performance of the developed control technique was evaluated with the conventional techniques and the results have revealed the effectuality of the proposed model in controlling conditions (normal, tumor and immune, cells). Here, the proposed method is compared with the conventional models such as Kalman Filter (KF), Extended Kalman filter (EKF) and Adaptive EKF. 


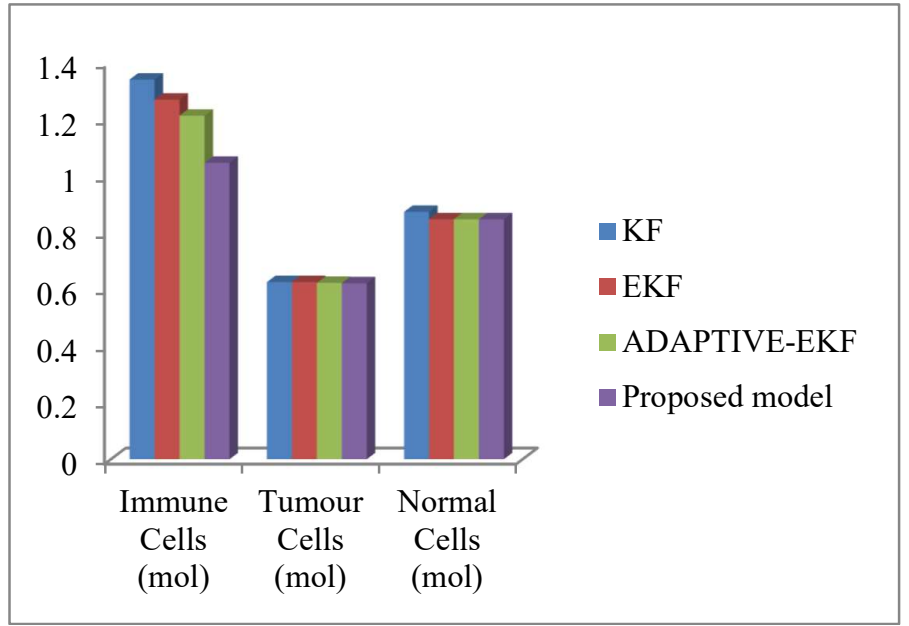

Fig. 1Performance analysis of the developed control theory and existing techniques

The completeanalysis of the developedmodelwith thetraditional techniques on tracking desired state values (normal, immune, anda tumor) is showninFig 1. In this analysis, developed control theory is established to possessobtainednearest desired values with existing techniques. Moreover, minimumcalculated values are attained by tumor cells, whichas wellshown tumor cells' non-existence. For Immune cells, the proposed method is $31 \%$ better than the KF, 28\% better than the EKF, and $23 \%$ better than the Adaptive EKF. The proposed method is 6\% better than the KF, $7.5 \%$ better than the $\mathrm{EKF}$, and $8 \%$ better than the Adaptive EKF for tumor cells. For Normal cells, the proposed method is $24 \%$ better than the KF, 21\% better than the EKF, and $18 \%$ better than the Adaptive EKF. Hence, superior parameter calculation is revealed to be obtainedusing the developed techniquewith the traditional techniques.

\section{Conclusion}

One of the main general cause for the psychological reactions was the Depression that patient's exhibit during diagnosis and treatment. In this work, a new robust controller was presented which impacts the dosage of drugs together with the estimation of the parameter. Moreover, a novel ASr-UKF was adopted as well an updating procedure was performed based upon prior error function, even if scaled to a nonlinear environment. Furthermore, for the updating procedure, a scaling factor was carried out that taken into consideration of historical error improvement. At last, the adopted controller was evaluated with the conventional models which show the drug dosage impact injection on immune normal, and tumor cells. Finally, the performance analysis of the proposed model exhibits its superiority over the conventional models

\section{Compliance with Ethical Standards}

Conflicts of interest: Authors declared that they have no conflict of interest.

Human participants: The conducted research follows the ethical standards and the authors ensured that they have not conducted any studies with human participants or animals.

\section{References}

[1] Salehoddin BouyaZeynab BarahoieFatemeh Kiani,"The effect of nursing self-care educational intervention on depression in women with breast cancer undergoing post-mastectomy chemotherapy: A quasi-experimental study", International Journal of Surgery OpenAvailable online 29 March 2021.

[2] X. Feng et al., "Accurate Prediction of Neoadjuvant Chemotherapy Pathological Complete Remission (pCR) for the Four Sub-Types of Breast Cancer," IEEE Access, vol. 7, pp. 134697-134706, 2019.

[3] M. Byra, K. Dobruch-Sobczak, Z. Klimonda, H. Piotrzkowska-Wroblewska and J. Litniewski, "Early Prediction of Response to Neoadjuvant Chemotherapy in Breast Cancer Sonography Using Siamese Convolutional Neural Networks," IEEE Journal of Biomedical and Health Informatics, vol. 25, no. 3, pp. 797-805, March 2021. 
[4] Florian MoikAlexander MakatsariyaCihan Ay,"Challenging anticoagulation cases: Cancer-associated venous thromboembolism and chemotherapy-induced thrombocytopenia - A case-based review of clinical management", Thrombosis Research, 4 January 2021.

[5] Amanda K. ArringtonChiu-Hsieh HsuTaylor S. Riall,"Survival After Margin-Positive Resection in the Era of Modern Chemotherapy for Pancreatic Cancer: Do Patients Still Benefit?", Journal of the American College of SurgeonsAvailable online 26 March 2021.

[6] H. Schattler, U. Ledzewicz, Optimal Control for mathematical models of cancer therapies, Springer, 2010.

[7] Reza Mohammadi AslYashar Shabbouei HaghHeikki Handroos,Adaptive square-root unscented Kalman filter: An experimental study of hydraulic actuator state estimation", Mechanical Systems and Signal Processing19 July 2019.

[8] H. Schattler, U. Ledzewicz, Optimal Control for mathematical models of cancer therapies, Springer, 2010.

[9] Dr.Sivaram Rajeyyagari,"Automatic Speaker Diarization using Deep LSTM in Audio Lecturing of e-Khool Platform",Journal of Networking and Communication Systems, vol 3, no. 4, October 2020.

[10] Suresh Babu Chandanapalli,Sreenivasa Reddy E,Rajya Lakshmi D,"Convolutional Neural Network for Water Quality Prediction in WSN",Journal of Networking and Communication Systems, vol 2, no 3, July 2019.

[11] Amol V Dhumane,"Examining User Experience of eLearning Systems using EKhool Learners", ",Journal of Networking and Communication Systems, vol 3, no 4, October 2020.

[12] Yongsheng Xu,"Hybrid GWO and CS Algorithm for UPQC Positioning in the Power Distribution Network", Journal of Computational Mechanics, Power System and Control, vol 3, no 3, July 2020 